\title{
Indícios da presença de fundamentos semióticos na literatura da ciência da informação
}

Indicios de la presencia de fundamentos semióticos en la literatura de la ciencia de la información

Indicia of the presence of semiotic foundations in the Information Science literature

\section{Carlos CÂNDIDO DE ALMEIDA}

Departamento de Ciência da Informação, Universidade Estadual Paulista (UNESP), Marília, SP. Brasil carlosalmeida@marilia.unesp.br

\begin{abstract}
Resumen
Las interfaces de la Semiótica con la Documentación en Brasil deben ser analizadas desde un punto de vista más amplio, buscando indicios de actividad interdisciplinar. Así, realizamos como complemento a un estudio teórico sobre los fundamentos semióticos de los procesos de análisis y de la mediación de la información, un análisis de cómo se están desarrollando los aspectos semióticos en la literatura sobre la Documentación. Para ello, se revisaron como fuentes los artículos, las actas de congresos y las tesis. Los resultados mostraron que los estudios semióticos relacionados con el análisis y tratamiento de la información y la mediación de la información se encuentran en un periodo inicial, especialmente en la literatura brasileña, a pesar de que se detecta un incremento en la cantidad de publicaciones.
\end{abstract}

Palabras clave: Semiótica. Documentación. Ciencia de la información. Estado de la cuestión. Relaciones interdisciplinares. Análisis bibliográfico. Brasil.

\section{Introdução}

A pesquisa "Fundamentos Semióticos da Análise e Mediação da Informação" teve como objetivo principal sistematizar as influências dos conceitos semióticos nos processos de análise documental de conteúdo e mediação da informação. A Semiótica é entendida ciência geral dos signos e entre seus protagonistas encontramos Charles Peirce e Ferdinand de Saussure. Contudo seu vínculo com outros campos fez surgiu uma miríade de tendências de investigação.

Inicialmente a proposta de pesquisa corroborou com os argumentos sobre a baixa relevância de publicações relativa à semiótica no campo da Ciência da Informação. O universo da pesquisa bibliográfica (corpus) constituiu-se de materiais cujos assuntos se correlacionam aos temas da pesquisa, divididos em artigos de periódicos; anais de eventos e monografias. A eleição das fontes de pesquisa relacionadas à Ciência da

\begin{abstract}
The interfaces between Semiotics of Information Science must also be analysed from a broader perspective, searching for signs of interdisciplinary involvement. So, as a complement to a theoretical study on the semiotic foundations of document analysis and information mediation, we undertook the analysis of the presence of semiotic perspectives in the Information Science literature -journals papers, conference proceedings and theses. The results showed that the semiotic studies related to the information analysis and information mediation are in an incipient stage, especially in Brazil, in spite of a substantial increase in the number of publications.
\end{abstract}

Keywords: Semiotics. Information science. Interdisciplinary relations. State of the art. Semiotics. Bibliographical analysis.

Informação foi orientada pelos temas análise da informação e mediação da informação.

$\mathrm{Na}$ sequência, relacionam-se os autores e os temas mais pesquisados que pontuam a interface entre os assuntos da pesquisa e a semiótica. O levantamento da bibliografia seguiu os seguintes procedimentos: localização dos periódicos, leitura do sumário, seleção dos artigos a partir dos títulos, leitura crítica do resumo, classificação e leitura dos materiais pelos integrantes do grupo de pesquisa. A busca dos títulos de periódicos foi realizada através da Base de Dados Referencial de Artigos de Periódicos em Ciência da Informação (BRAPCI), buscando as publicações dos periódicos da área da Ciência da Informação nacionais, desde a primeira edição até o número do primeiro semestre de 2013. Um outro critério foi a inclusão no ranking Qualis para periódicos, selecionando os títulos compreendidos entre conceito Qualis A1 e B2. Os títulos consultados foram: Trans/nformação, 
Brazilian Journal of Information Science, Informação e Sociedade: Estudos, Revista da Escola de Biblioteconomia da Universidade Federal de Minas Gerais, Informação \& Informação, Perspectivas em Gestão \& Conhecimento, Liinc em Revista, Em Questão: Revista da Faculdade de Biblioteconomia e Comunicação da Universidade Federal do Rio Grande do Sul, Perspectivas em Ciência da Informação, InCID: Revista de Ciência da Informação e Documentação, Revista Digital de Biblioteconomia e Ciência da Informação, Biblionline, Ciência da Informação, Encontros Bibli, Revista Brasileira de Biblioteconomia e Documentação, DataGramaZero e Comunicação \& Informação.

Alguns títulos foram acrescentados ao número inicial, segundo o planejamento da metodologia do projeto. Esta decisão foi necessária para dar mais abrangência às fontes pesquisadas. No que se refere aos artigos de periódicos internacionais indicados na metodologia do projeto optou-se pelo tratamento em pesquisa posterior em razão do número de fontes e materiais levantados para esta pesquisa em âmbito nacional. Os periódicos internacionais em Ciência da Informação (Journal of documentation, Information Processing and Management, Knowledge Organization, Scire: Organización y Representación del Conocimiento, Anales de Documentación, Bulletin of the American Society for Information Science and Technology, Journal of the American Society for Information Science, Library Trends, Information Research: an international electronic journal), bem como o periódicos em Filosofia e Semiótica (Cognitio: Revista de Filosofia, Trans/ Form/ Ação, Cognitio-Estudos: Revista Eletrônica de Filosofia, Applied Semiotics / Sémiotique appliquée, Journal Semiotics, Evolution, Energy, and Development e Semiotica) serão objeto de análise em pesquisa futura.

Com os anais de eventos, adotaram-se as mesmas estratégias que o levantamento dos artigos de periódicos. Os materiais de eventos consultados foram os anais do Encontro Nacional de Pesquisa em Ciência da Informação (ENANCIB), os anais de eventos da International Society for Knowledge Organizational (ISKO), notadamento os capítulos Espanhol $(1993,1995,1997,1999,2001,2005,2007$, 2009), Francês (2007, 2009), Canadence (2007, 2009, 2011, 2013), Brasileiro (2012, 2013), e os da International Conference on Conceptions of Library and Information Sciences (CoLIS), nas edições 5, 6, 7 e 8. Alguns capítulos da ISKO, bem como alguns anais da COLIS, não foram obtidos para esta análise, o que deverá ser objeto de pesquisa posterior.
As monografias (teses e as dissertações) foram identificadas pela consulta às bases Banco de Teses da Coordenação de Aperfeiçoamento de Pessoal de Nível Superior (CAPES), DART Europe e Networked Digital Library of Theses and Dissertations (NDLTD) por meio dos termos relacionados a pesquisa (ciência da informação e semiótica), no período de 1900 a 2012. A seleção dos textos monográficos encontrados se deu pela análise do título, do abstract e das palavras chave, com fundamento no grau de proximidade das temáticas do projeto. Buscouse assim inferir se os temas discorridos em cada monografia eram de algum modo análogo ao tema do projeto em três níveis: identificação temática (se o tema, em seus traços essenciais, se assemelhava ao projeto ou se apresentava aspectos temáticos solidários e tangenciais); proximidade metodológica (se cada monografia utilizava a semiótica em função de expediente metodológico); e idiomático (selecionaram-se apenas textos monográficos em espanhol, francês, inglês, italiano e português; tendo em conta que foram recuperados também textos em alemão, grego, chinês, dinamarquês, entre outros).

Do ponto de vista da interpretação dos resultados, ressalta-se que esta pesquisa não se propôs a estabelecer uma estimativa quantitativa dos autores e temas mais pesquisados e onde se encontram publicados. Contudo, tal tipo tratamento dos dados foi necessário para levantar indícios da abordagem do tema. O fundamental no relatório completo da pesquisa, da qual esta comunicação é um apêndice, foi discutir os avanços teóricos que os conceitos podem representar para explicar temas associados à análise documental e mediação da informação, o que se acredita foi alcançado com os capítulos anteriores.

As análises a seguir permitem apontar alguns indícios e vestígios da perspectiva semiótica, embora pouco difundida, parece-se em crescente desenvolvimento na área.

\section{Indícios da Presença do Tema Semiótica na Literatura da Área}

No que se refere aos periódicos científicos nacionais da Ciência da Informação levantados no período de coleta de materiais analisados pelo grupo de pesquisa, apresentam-se a seguir os autores indicados. A lista com os autores mais citados foi produzida partindo dos artigos recuperados no levantamento junto aos periódicos, verificando-se as referências de cada um dos 21 artigos recuperados com temáticas afins à pes- 
quisa, os quais registraram o total de 319 autores citados.

Desses autores, os mais citados foram Peirce (24 citações ou 7,52\%), Lara (24 citações ou $7,52 \%$ ) e Santaella (23 citações ou $7,21 \%$ ). As referências em que não se trata de autor, como normas, por exemplo, optou-se por classificá-los como autor-entidade, estes foram citados 20 vezes. Entre os demais autores, quatro foram citados 9 vezes; dois foram citados 8 vezes; dois foram citados 7 vezes; dois foram citados 6 vezes; seis foram citados 5 vezes; oito foram citados 4 vezes; onze foram citados 3 vezes; trinta e seis foram citados 2 vezes; e 235 foram citados uma única vez. Dessa forma, estão expostos os dados no gráfico a seguir para melhor visualização dos dados.

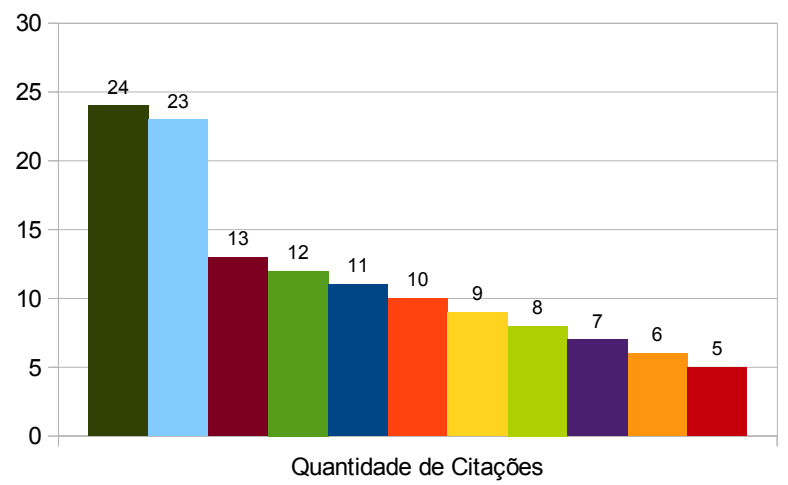

- Peirce, Lara
- Santaella
- Deleuze, Kobashi
= Eco, Tálamo
- Almeida
- Silveira
- Lévy, Mai, Moura,
Nöth
n Guimarães, Pinto
- Cintra, Dahlberg
- Barreto, Monteiro
- Cunha, Fujita, Gardir
Guattari, Lancaster,
Thellefsen

Gráfico 1. Autores mais citados em artigos dos periódicos nacionais em Ciência da Informação

Um conjunto de oito autores foi citado 4 vezes e um grupo de onze autores foi citado 3 vezes nos artigos selecionados. Os demais autores citados 2 ou uma única vez não foram considerados neste gráfico. Nesse sentido, pode-se concluir que os primeiros cinco autores mencionados referem-se às temáticas da Semiótica geral e da aplicação da semiótica no campo da organização do conhecimento, haja vista a presença dos autores deste campo, como Lara, Tálamo, Kobashi e Almeida. Por outro lado, constatamse também a presença de autores bem citados que versão sobre semiótica geral, é o caso de Peirce, Eco, Nöth e Santaella.

Entre os temas mais citados, os quais estão relacionados ao eixo da pesquisa organização da informação e do conhecimento pode-se citar, além de semiótica, organização da informação e do conhecimento, linguagem documentária e análise documentária, ciência da informação, indexação e análise de assunto. Foram recuperados 21 artigos, tratando da semiótica relacionada aos temas da pesquisa. Constata-se que o tema de maior recorrência é a conexão entre semiótica e organização da informação e do conhecimento.

O tema semiótica e organização da informação e da conhecimento surge com 9 (42,85\%), semiótica e linguagem documentária/análise documentária, com $6(28,57 \%)$, semiótica e ciência da informação, com $3(14,28 \%)$, semiótica e indexação, com 2 (9,52\%) e semiótica e análise de assunto, com apenas 1 trabalho (4,76\%).

Considerando o resultado de 21 artigos, abordando os temas expostos anteriormente nos artigos recuperados, o periódico com maior número de trabalhos foi Encontros Bibli, com 5 trabalhos $(23,80 \%)$, seguido de Informação e Sociedade, com 4 artigos (19,04\%).

\begin{tabular}{|c|c|c|}
\hline Periódico & $n .^{\circ}$ & $\%$ \\
\hline TransInformação & 1 & 4,76 \\
\hline Brazilian Journal of Information Science & 0 & - \\
\hline Informação e Sociedade: Estudos & 4 & 19,04 \\
\hline $\begin{array}{l}\text { Revista da Escola de Biblioteconomia da } \\
\text { Universidade Federal de Minas Gerais }\end{array}$ & 0 & - \\
\hline Informação \& Informação & 2 & 9,52 \\
\hline Perspectivas em Gestão \& Conhecimento & 0 & - \\
\hline Liinc em Revista & 1 & 4,76 \\
\hline $\begin{array}{l}\text { Em Questão: Revista da Faculdade de } \\
\text { Biblioteconomia e Comunicação da } \\
\text { Universidade Federal do Rio Grande do Sul }\end{array}$ & 0 & - \\
\hline Perspectivas em Ciência da Informação & 1 & 4,76 \\
\hline $\begin{array}{l}\text { InCID: Revista de Ciência da Informação e } \\
\text { Documentação }\end{array}$ & 0 & - \\
\hline $\begin{array}{l}\text { Revista Digital de Biblioteconomia e Ciência } \\
\text { da Informação }\end{array}$ & 1 & 4,76 \\
\hline Biblionline & 0 & - \\
\hline Ciência da Informação & 3 & 14,28 \\
\hline Encontros Bibli & 5 & 23,80 \\
\hline $\begin{array}{l}\text { Revista Brasileira de Biblioteconomia e } \\
\text { Documentação }\end{array}$ & 0 & - \\
\hline DataGramaZero & 3 & 14,28 \\
\hline Comunicação \& Informação & 0 & - \\
\hline Total & 21 & 100 \\
\hline
\end{tabular}

Diversos periódicos da área ainda não publicaram nenhum trabalho sob o tema semiótica. Os que assim fizeram trataram especialmente da temática da organização da informação e organização do conhecimento. Nesse sentido, um desdobramento semiótico da mediação parece 
não encontrar uma consciência possível, tal como preconizou Goldmann.

Sobre o tema da mediação foram obtidos 50 artigos, o maior número deles publicados em 2009 (6 artigos ou 12\%) e 2004 (5 artigos ou $10 \%$ ). A primeira publicação com a temática presente nos periódicos nacionais em Ciẽncia da Informação é de 1990. Foram citados nos artigos selecionados 89 autores, destes alguns se destacam. Almeida Júnior com 13 citações (28,3\%), autores com 3 citações cada ou $6,5 \%$ (Alcará, Barros, Castro, Grogan, Henriques, Kuschnir, Oliveira, Figueiredo) e autores com 2 citações $(4,3 \%)$ cada (Baptista, Barreto, Barros, Barros, Bellotto, Café, Carvalho, Côrtes, Costa, Cunha, Dias, Ferneda, Figueiredo N, Fraiz, Francisco, Kurtz, Menezes, Novelli). 62 autores foram citados uma única vez.

Entre os trabalhos levantados nenhum fez relação da mediação com a Semiótica. Temas como mediação da informação, do ponto de vista conceitual, acesso à informação, usuários da informação e serviços de referência foram muito citados na literatura, contudo, a interface com a semiótica não foi observada. O maior número de artigos foi publicado pela revista Ciência da Informação (com 5 artigos), seguida pelas revistas Perspectivas em Ciência da Informação e DataGramaZero, com 3 artigos cada.

A análise destes dados sugere que o tema mediação não está sendo explorado do ponto de vista simbólico. Esse viés é expressivo no campo da organização da informação e do conhecimento, o que mostra que os fundamentos semióticos, notadamente os conceitos da semiótica de Peirce estão sendo utilizados para consolidar o campo, abrindo uma nova fronteira de estudos. O que não pode ser afirmado a respeito do campo da mediação da informação.

\begin{tabular}{lccccc}
\hline Temas & \multicolumn{5}{c}{ COLIS } \\
\hline & 5 & 6 & 7 & 8 & $T$. \\
\hline Acesso à informação & 1 & 0 & 1 & 0 & 2 \\
\hline Arquitetura da informação & 0 & 0 & 0 & 1 & 1 \\
\hline Arquivo digital & 0 & 2 & 0 & 1 & 1 \\
\hline Arquivologia & 0 & 0 & 0 & 1 & 1 \\
\hline Arquivos e bibliotecas digitais & 0 & 0 & 0 & 1 & 1 \\
\hline Bibliometria & 3 & 0 & 1 & 3 & 7 \\
\hline Biblioteca digital & 1 & 0 & 0 & 3 & 6 \\
\hline Biblioteconomia & 0 & 4 & 2 & 17 & 23 \\
\hline Bibliografia & 0 & 1 & 1 & 0 & 2 \\
\hline Busca informacional & 0 & 1 & 0 & 0 & 1 \\
\hline
\end{tabular}

\begin{tabular}{|c|c|c|c|c|c|}
\hline Bibliotecas e arquivos & 0 & 0 & 1 & 0 & 1 \\
\hline Ciência da Informação e educação & 0 & 16 & 1 & 4 & 21 \\
\hline Colaboração científica & 0 & 0 & 1 & 0 & 1 \\
\hline Comportamento informacional & 1 & 7 & 5 & 3 & 16 \\
\hline Comunicação & 0 & 0 & 0 & 1 & 1 \\
\hline Conceito de informação & 1 & 0 & 0 & 0 & 1 \\
\hline Descrição documental & 0 & 0 & 1 & 0 & 1 \\
\hline Epistemologia & 0 & 8 & 3 & 9 & 20 \\
\hline E-social Science & 1 & 0 & 0 & 0 & 1 \\
\hline Estudo de usuários & 1 & 1 & 0 & 0 & 2 \\
\hline Estudos de autoria & 0 & 0 & 0 & 1 & 1 \\
\hline Estudos de gênero & 0 & 0 & 0 & 1 & 1 \\
\hline Gestão do conhecimento & 0 & 1 & 0 & 0 & 1 \\
\hline Gestão da informação & 0 & 0 & 0 & 1 & 1 \\
\hline Informação biomédica & 0 & 1 & 0 & 0 & 1 \\
\hline Informação e minorias & 0 & 0 & 0 & 1 & 1 \\
\hline Informação oral & 0 & 1 & 1 & 0 & 2 \\
\hline Information literacy & 0 & 0 & 3 & 7 & 10 \\
\hline Linguagem computacional & 0 & 0 & 1 & 0 & 1 \\
\hline Mediação da informação & 0 & 1 & 0 & 0 & 1 \\
\hline Meta-análise e meta-síntese & 0 & 0 & 1 & 0 & 1 \\
\hline Necessidade informacional & 0 & 0 & 1 & 0 & 1 \\
\hline Necessidade e relevância da inf. & 1 & 0 & 0 & 0 & 1 \\
\hline Organização do conhecimento & 0 & 0 & 0 & 3 & 3 \\
\hline Práticas de publicação & 0 & 0 & 0 & 1 & 1 \\
\hline Produtos de informação & 0 & 1 & 0 & 0 & 1 \\
\hline Recuperação da informação & 5 & 1 & 0 & 1 & 7 \\
\hline Redes sociais & 0 & 0 & 0 & 1 & 1 \\
\hline Repositórios digitais & 1 & 0 & 0 & 0 & 1 \\
\hline Serviço de informação & 0 & 0 & 0 & 1 & 1 \\
\hline Sistema de informação & 1 & 2 & 0 & 0 & 3 \\
\hline Tecnologia da informação & 0 & 2 & 0 & 0 & 2 \\
\hline Web & 0 & 0 & 0 & 1 & 1 \\
\hline Wikipedia & 0 & 0 & 1 & 0 & 1 \\
\hline
\end{tabular}

Tabela 2. Número de trabalhos por temas nos Colis $5,6,7$ e 8

No que se referem aos anais de eventos, apresenta-se a na sequência o levantamento realizado junto ao COLIS. Das oito reuniões da COLIS, foram encontrados todos os textos referentes às quatro últimas edições. Os textos das quatro primeiras ainda não foram adquiridos e por esta razão não foram inseridos nesta análise. Considerando o total de trabalhos, foram apresentados nas oito edições do COLIS 286 trabalhos. Contudo, como nem todos puderam 
ser recuperados, o tratamento dos dados centrou-se apenas nas edições COLIS 5, com 22 papers, COLIS 6 com 53 papers, COLIS 7 com 26 papers e COLIS 8 com 69 papers. As buscas para localização dos trabalhos do COLIS foram realizadas por meio dos bancos de dados Emerald e Portal de Periódicos da CAPES. No entanto, a quase totalidade dos textos foi encontrada por meio de motores de busca. Entre os assuntos tratados nos eventos, pode-se perceber a incidência de temas relacionados às questões da linguagem. Os temas mais discutidos, considerando as apresentações de trabalhos nos anais identificados, foram, em primeiro lugar, Biblioteconomia, em segundo lugar, Ciência da Informação e Educação, e em terceiro lugar, Epistemologia. Tanto mediação quanto análise do conteúdo da informação tiveram poucos trabalhos publicados no estrato analisado. A tabela 2 ilustra a posição subalterna de tais temas nas edições do evento.No que tange os trabalhos publicados nos eventos promovidos pela ISKO também se constata uma baixa incidência de trabalhos cujos temas versam a semiótica, embora a correção entre semiótica e organização da informação seja bem relevante. Os dados representados foram analisados a partir da leitura de resumos, títulos e palavraschave de artigos da ISKO contidos nos capítulos espanhol, francês, canadense e brasileiro, referentes aos termos pesquisados (Linguagem, Mediação da Informação, Sistemas de Informação e Semiótica). Dos termos buscados houve ainda o acréscimo de expressões como organização do conhecimento, representação do conhecimento, mediação do conhecimento, linguagens documentais e ontologias semióticas. Considerando os trabalhos levantados, encontraram-se um total de 474 trabalhos nos diferentes anais da ISKO. Contudo, selecionando os trabalhos relacionados à pesquisa, chegou-se a um número de 20 trabalhos, isto é, $4,21 \%$ do total. Os temas mais recorrentes nos artigos são organização do conhecimento e representação do conhecimento. Não obstante, considera-se como fundamental aprofundar a análise dos trabalhos da ISKO recuperados, não com vistas a indicar a quantidade de temas, mas para discutir o impacto dos conceitos na teoria da organização do conhecimento. Parte destas ideias foram apresentadas no capítulo que tratou dos fundamentos semióticos na análise da informação.

Com respeito aos trabalhos do Enancib, foram levantados materiais de 13 edições, relativas aos congressos 1994, 1995, 1997, 2000, 2003, 2005, 2006, 2007, 2008, 2009, 2010, 2011 e 2012, totalizando 2130 trabalhos publicados. Desde, apenas $61(2,86 \%)$ trabalhos foram se- lecionados pois trataram especificamente dos termos de busca linguagem, mediação da informação, semiótica, organização do conhecimento, linguística documentária, terminologia, conceitos linguísticos, ciência da informação e epistemologia. Essa diversidade temática ainda não significou uma grande monta de trabalhos que expressem, por exemplo, uma corrente teórica sobre semiótica no campo da Ciência da Informação no Brasil.

Nesse universo de 61 trabalhos, os autores que mais se destacaram foram Moura, Almeida e Gomes, com 4 trabalhos cada um, seguidos de Grippa com 3 trabalhos. Os demais autores computaram dois trabalhos. Os demais 58 autores recuperados contribuiram com apenas um trabalho. É possível observar também uma tendências dos trabalhos nos últimos anos, notando um avanço no número de trabalhos publicados a partir de 2007.

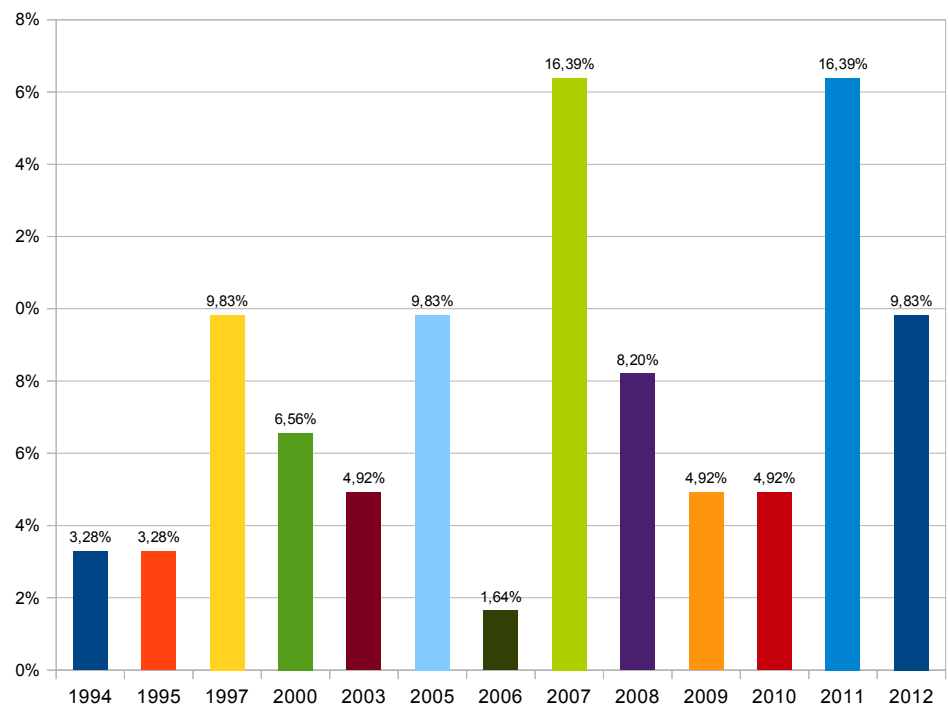

Gráfico 2. Distribuição dos trabalhos nas edições do Enancib

Observando a distribuição dos temas, verificase que o maior número de trabalho está concentrado no tema mediação com $44,26 \%$, enquanto o tema semiótica corresponde a $13,11 \%$ e linguagem com $6,56 \%$.

As monografias também foram objeto do levantamento da pesquisa. Como mencionado anteriormente, foram recuperados um total de 195 materiais e selecionados $53(27,17 \%)$ a partir do levantamento nas bases DART Europe e NDLTD e considerando as especificidades da pesquisa. Destes materiais, 42 são teses e 11 são dissertações e a maior parte defendida nos Estados Unidos e Canadá. A tabela a seguir 
ilustra a disposição das monografias por países de origem.

\begin{tabular}{lll}
\hline Países & $\mathrm{N}^{\circ}$ & $\%$ \\
\hline Estados Unidos & 18 & 33,96 \\
\hline Canadá & 11 & 20,75 \\
\hline Brasil & 7 & 13,20 \\
\hline Austrália & 4 & 7,54 \\
\hline França & 3 & 5,66 \\
\hline Suécia & 2 & 3,77 \\
\hline Estônia & 1 & 1,88 \\
\hline Alemanha & 1 & 1,88 \\
\hline Espanha & 1 & 1,88 \\
\hline Estônia & 1 & 1,88 \\
\hline Grécia & 1 & 1,88 \\
\hline Itália & 1 & 1,88 \\
\hline Nova Zelândia & 1 & 1,88 \\
\hline Suíça & 1 & 1,88 \\
\hline Total & 53 & 100 \\
\hline
\end{tabular}

Tabela 3. Países de origem das monografias

\begin{tabular}{ll}
\hline Autores & $N^{\circ}$ \\
\hline Peirce, C.S. & 17 \\
\hline Halliday, M.A.K. & 15 \\
\hline Lotman, J. & 14 \\
\hline Eco, U. & 10 \\
\hline Queiroz, J. & 10 \\
\hline Salthe, S. N. & 10 \\
\hline Martin, J.R. & 9 \\
\hline Poynton, C. & 7 \\
\hline Maturana, H. & 6 \\
\hline Houser, N. & 6 \\
\hline Jackson, B. S. & 6 \\
\hline Deely, J. & 5 \\
\hline Bohm, D. & 5 \\
\hline Foucault, M. & 5 \\
\hline Gräzin, I. & 5 \\
\hline Hulswit, M. & 5 \\
\hline Kevelson, R. & 5 \\
\hline Nöth, W. & 5 \\
\hline Short, T. L. & 5 \\
\hline Wish, M. & 5 \\
\hline
\end{tabular}

Tabela 4. Autores mais citados no tema Semiótica em monografias
Em razão dos países citados houve uma maior concentração de materiais de língua inglesa (41 monografias), seguida do português (7 monografias), francês (3 monografias), espanhol e italiano (1 monografia cada). Em relação aos temas principais, apenas 5 teses $(9,43 \%)$ abordaram especificamente o tema semiótica. Tais temas foram identificados a partir da leitura dos resumos (abstract) e constituem, cada qual, a forma temática mais genérica encontrada. Observe-se que em todas as monografias há o recurso à semiótica, mas nem os trabalhos a tomam por escopo. Por esta razão somente as que versam essencialmente sobre Semiótica são as que aqui foram categorizadas no âmbito do tópico semiótica. Especificamente nas monografias que tratam do tema semiótica, podese constatar a relevância de Peirce, bem como a incidência de citações de outros teóricos conhecidos da semiótica, como Lotman e Eco.

\section{Considerações finais}

Em síntese, com o levantamento realizado pode-se concluir que os estudos semióticos relacionados à análise da informação e mediação da informação são incipiente de um ponto de vista meramente quantitativo, especialmente na literatura nacional. A despeito de os problemas semióticos estarem presentes no momento da interação social da mediação institucionalprofissional, a questão simbólica ainda não é significante na literatura da mediação nos estudos da Ciência da Informação.

Considera-se que os objetivos da pesquisa com o levantamento foram alcançados, e refutou-se a ideia de qua a literatura especializada consultada tem uma consciência semiótica sobre os problemas da mediação e apropriação da informação. Contudo, sublinha-se a necessidade de realizar estudos teóricos-conceituais para explicar o processo de apropriação da informação sob um olhar semiótico. A porcentagem de materiais selecionados para uma análise das citações, dos autores e temas nas fontes pesquisadas ainda é muito baixa em relação a outras temáticas, o que significa que a prioridade ainda está em temas e objetos de estudos mais aplicados e que tenham demonstrado eficiência em explicações anteriores. Considerando os trabalhos do ENANCIB, apenas $2,86 \%$ foi selecionado por tratar de temáticas conexas, e destes apenas um grupo de três autores apresentou uma continuidade no tratamento dos temas. Com respeito às monografias, a maioria produzida em inglês está comprometida com uma discussão semiótica, ressaltando-se a influência da linha peirceana comprovada pelo número de citações. Na mesma direção, é possível perce- 
ber a influência da abordagem peirceana nos artigos de periódicos nacionais selecionados.

No que tange aos eventos, pode-se verificar que a ISKO ainda representa o maior número de trabalhos no campo da análise da informação e organização do conhecimento, em função do propósito desta comunidade científica. Não obstante, os anais do COLIS recuperados deram a dimensão do impacto dos estudos de mediação e análise da informação diante dos demais temas da Ciência da Informação, sendo minoritários em relação às questões conceituais.

Esta breve exposição, se não pode concluir favoravelmente sobre os fundamentos semióticos nos processos de análise e mediação da informação, pode sustentar que este tema está sendo aplicado com mais intensidade nos últimos anos. Contudo, a análise qualitativa destes materiais poderá revelar por que certos conceitos semióticos são utilizados para explicar problemas de informação, e não apenas restringir à dentificação de conceitos na literatura da área. Nesse sentido, projeta-se a continuidade da pesquisa produzindo interpretações a respeitoda interdisciplinaridade entre Semiótica e Ciência da Informação.

\section{Agradecimentos}

Agradeço ao auxílio recebido pelo Conselho de Desenvolvimento Científico e Tecnológico (CNPq) para o desenvolvimento desta pesquisa (Processo: 475332/2011-3). Ademais, gostaria de agradecer a colaboração de Mona Cleide Quirino Farias, Rafael Semidão, Márcio Ferreira da Silva, Viviane Oliveira e Aline Reis, do grupo de pesquisa Fundamentos Teóticos da Informação.

\section{Referências}

Almeida, C. C. (2011). Sobre o pensamento de Peirce e a organização da informação e do conhecimento. // Liinc em Revista. 7:1 (2011) 104-120.

De Wall, C. (2007). Sobre pragmatismo. São Paulo: Loyola, 2007.

Friedman, A.; Thellefsen, M. (2011). Concept theoty and semiotics in knowledge organization. // Journal of Documentation. 67:4 (2011) 644-674.

Mai, J-E. (2001). Semiotics and indexing: an analysis of the subject indexing process.// Journal of Documentation. 57:5 ( 2001 ) 591-522.

Marafioti, R. (2005). Charles S. Peirce: el éxtasis de los signos. Buenos Aires: Biblos, 2005.

Peirce, C. S. (2000). Semiótica. 3. ed. São Paulo: Perspectiva, 2000.

Thellefsen, T. L.; Thellefsen, M. M. (2004). Pragmatic semiotics and knowledge organization. // Knowledge Organization. 31: 3 (2004) 177-187.

Enviado: 2014-04-25

Aceptado: 2014-06-16. 
